\title{
160-min OSCILLATIONS OF THE SUN AS THE MEAN OF STUDY OF ITS INTERNAL STRUCTURE*
}

\author{
E. A. GAVRYUSEVA, YU. S. KOPYSOV, and G. T. ZATSEPIN \\ Institute for Nuclear Research of the Academy of Sciences of the USSR, \\ 60th October Anniversary prospect, 7a, Moscow 117312, U.S.S.R.
}

\begin{abstract}
The investigation of the models of the contemporary Sun with a mixed core has shown that the amplitude of some gravity modes of oscillations of the star can be mainly concentrated in the central region. This phenomenon takes place if the node of the amplitude of radial displacement coincides with the boundary of the mixed core. In this case the core can be regarded as a driving generator of the oscillations, determining their period and phase. It is suggested as the explanation of the observational properties of the 160 -min oscillation.
\end{abstract}

\section{Introduction}

In recent years there have become available new observational data in the solar physics. However such an important information about the Sun as the low high-energy neutrino flux (Davis, 1978) and the pulsation of the solar surface with 160-min period (Kotov et al., 1976; Brookes et al., 1976) did not find well grounded theoretical explanations. The radial oscillations can not have such long period. The nonradial oscillations with high spheroidal number $l$ and low odd $l=1,3$ could not be registered in the experiment of Severny (Severny et al., 1979). In the spectrum of quadrupole oscillations only moderately high order gravity $g$-modes correspond to 160 -min pulsation. In this case according to usual theoretical considerations it is impossible to understand why the lower modes are not observed though they must be get excited first of all.

The wonderful property of 160 -min pulsation consist in their possibility sometimes to disappear and then to appear again with the same phase as if they had not disappeared at all (Severny et al., 1979). This property allowed to assume this oscillation to be concentrated in the inner regions of the Sun (Zatsepin et al., 1980; Gavryuseva and Kopysov, 1981). In standard models of the Sun the amplitude of displacements is higher in the envelope than in the internal region. The standard models of the Sun have also difficulties in the interpretation of the other experimental data. For example the calculated flux of high energy solar neutrino is greater than observed by Davis et al. (1978). It is worth mentioning that the bulk experimental data about the Sun are connected to the contemporary state of the Sun. Therefore, there is sense to construct such models of the contemporary Sun which would be in accordance with the experimental results. In the models of the contemporary Sun with the mixed core the high energy neutrino flux is lower than in the standard models.

\footnotetext{
* Proceedings of the 66th IAU Colloquium: Problems in Solar and Stellar Oscillations, held at the Crimean
} Astrophysical Observatory, U.S.S.R., 1-5 September, 1981. 
A great number of models of the contemporary Sun with various surface hydrogen abundances $X_{0}$ and with the different parts of mixed mass $M_{\text {mix }}$ has been constructed (Gavryuseva, 1981). The core hydrogen abundance $X_{c}$ for each model has been accepted constant throughout up to the core boundary and then increased up to $X_{0}$ as

$$
X(M)=X_{0}+\left(X_{c}-X_{0}\right) \exp \left\{-\left(\frac{M-M_{\text {mix }}}{\Delta M}\right)^{2}\right\}
$$

where $\Delta M$ is the thickness of the transitional layer.

This article devotes to investigation of these models from helioseismological point of view.

\section{Results}

The small linear adiabatic oscillations have been studied originaly within the framework of multizone polytropic models (Zatsepin et al., 1980; Gavryuseva and Kopysov, 1981) and later on models with different parts of the mixed mass $M_{\text {mix }}$ for series of hydrogen concentrations in the envelope $X_{0}$ and thickness of the transitional layer $\Delta M$.

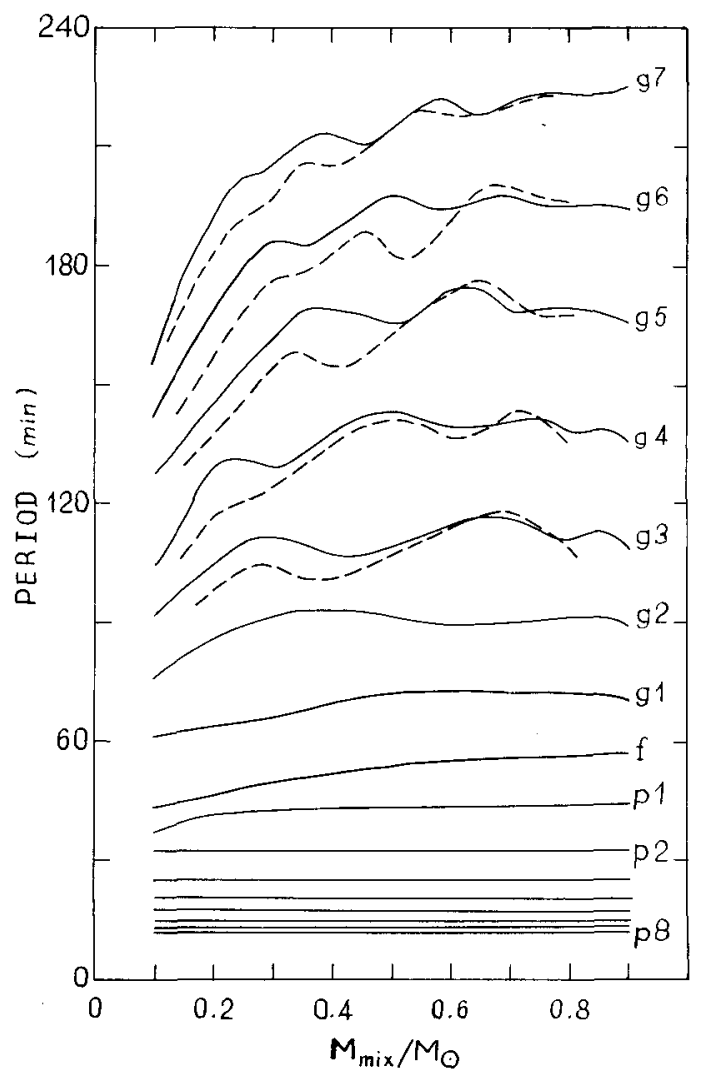

Fig. 1. The periods of quadrupole oscillations of the Sun as a function of the mixed core size for the envelope hydrogen abundance $X_{0}=0.7$ (continuous line) and $X_{0}=0.75$ (broken line). 
The radial oscillations are practically not sensitive to variations of the parameters $X_{0}$ and $M_{\text {mix }}$. They actually occupy only the very upper layers of the Sun. In all models the amplitude of the radial displacement on the surface exceeds the maximum displacement in the central zones by 3 to 4 orders of magnitude. These oscillations have periods less or about one hour. Because of that they can not be used to explain the 160 -min oscillation. The quadrupole oscillations are of two kinds. Acoustic modes have low periods. The behaviour of their eigenfunctions is similar to radial ones. The frequency and the form of eigenfunctions of long-periodical gravity modes are conditioned by structure of the Sun as a whole. Periods of $g$-modes of quadrupole oscillations, presented in Figure 1, are connected very closely with the values of $M_{\text {mix }}$ parameter. The greater $M_{\text {mix }}$ the longer the periods of gravity modes, especially of the high order ones. For $M_{\text {mix }}$ smaller than $0.25 M_{\odot}$ the 160 -min period will belong to $g 6, g 7$ and so on by suitable selection of $X_{0}$. For values of $M_{\text {mix }}$ in interval from $0.25 M_{\odot}$ to $0.5 M_{\odot}$ the hydrogen concentration in envelope can be selected so that the obtained model will have oscillations with 160-min periods which are identified as $g 5$-mode. For $M_{\text {mix }}>0.5 M_{\odot}$ at any $X_{0}$ the $g 5$-mode has a period some more than 160 -min but the $g 4$-mode has a
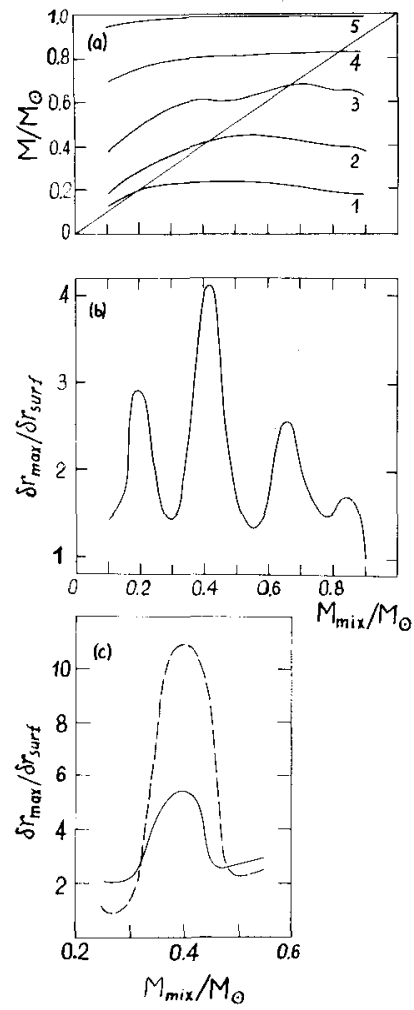

Fig. 2. (a) Position of the nodes of the radial displacement amplitude and (b) the ratio $\delta r_{\max } / \delta r_{\text {surf }}$ for g5-mode of quadrupole oscillations of the Sun as a function of the mixed core size at $X_{0}=0.7$, $\Delta M=0.05 M_{\odot}$. (c) The same as (b) at $X_{0}=0.75, \Delta M=0.05 M_{\odot}$ (continuous line) and $\Delta M=0.01 M_{\odot}$ (broken line). 
period less than that. The shape of eigenfunction of radial displacement of $g$-modes has proved to depend on the size of the mixed core. It is seen from Figure $2 b$, in which the ratio of the maximal amplitude of radial displacement inside the core $\delta r_{\max }$ for the g5-mode of quadrupole oscillations to that in the external layers $\delta r_{\text {surf }}$ ( $\delta r_{\text {surf }}$ is usually maximal on the surface) is plotted as a function of $M_{\text {mix }}$ for $X_{0}=0.7$. It is important to note that this ratio gets a maximum every time as some internal node finds itself at the boundary of the mixed core as seen from the comparison between Figures $2 \mathrm{a}$ and $2 b$. The behaviour of the positions of the nodes of the radial displacement amplitude by change $M_{\text {mix }}$ is shown in Figure 2a. The more the difference in hydrogen concentration in core and in envelope and the thinner the transition layer $\Delta M$ the stronger this effect is expressed. Figure $2 \mathrm{c}$, which shows the dependence of the ratio of $\delta r_{\text {max }}$ to $\delta r_{\text {surf }}$ of $g 5$-mode on the size of the transition layer $\Delta M$ at $X_{0}=0.75$ and its comparison with Figure $2 b$ confirm that.

This property of the amplitude of displacement allows to understand why only one of the high order $g$-modes of nonradial oscillations can be observed while the lower order modes can be absent. We suppose the losses of oscillation energy conditioned mainly by the viscous forces are most considerable in the convective envelope. These losses are proportional to the square of the amplitude displacement. If the core is a driving generator of the oscillations and their amplitude is great only there they will feebly damped in the external layer, because the dissipation of the oscillation energy will be weak thanks to small displacement. The more the amplitude in the internal regions of the Sun exceeds the amplitude on the surface the more probable is excitation of the oscillations in the core, which is due to thermonuclear driving proposed by Dilke and Gough (1972) (see also Christensen-Dalsgaard et al., 1974).

On inspecting Figures 1 and $2 \mathrm{~b}$ one can conclude that the mixed core could become a wave trap for the $g 5$-mode of quadrupole oscillations only provided $M_{\text {mix }} \simeq 0.4 M_{\odot}$. If one considers a model with $M_{\text {mix }}=0.4 M_{\odot}$ as a model of the contemporary Sun so, besides the $g 5$-mode, the generation of the 87 -mode with the period about $205 \mathrm{~min}$, depending on $X_{0}$, is possible. $g 6, g 4$ and lower modes will be subject to strong dissipations in the external layer, because the amplitude of the radial displacement does not decrease but it increases to the surface.

In this model capture rate of the solar neutrino in a ${ }^{37} \mathrm{Cl}$ detector is changing from $\approx 3 \mathrm{SNU}$ to $6.3 \mathrm{SNU}$ for $\mathrm{S}_{34}(0)=0.29-0.67 \mathrm{keV}$-barns.

The 160-min oscillation will be underlined also at $l=2$ for $g 7$-mode if mixed core occupies 0.1 solar mass, but the model of the Sun with $M_{\text {mix }}=0.1 M_{\odot}$ has very great high energy neutrino fluxes.

\section{Conclusion}

As has been shown above the solar interiors may become a selective trap for some gravity modes. This can take place if the Sun has a mixed core and the radial amplitude of the oscillation has the node at its boundary. In this case the oscillations of the Sun could be driven by nuclear reactions because of the radial amplitudes of the trapped 
mode are maximum inside the core. The explanation of properties of 160 -min oscillation could come out in models of the contemporary Sun with the mixed mass equal to $M_{\text {mix }} \simeq 0.4 M_{\odot}$. Their preferable exitation could be explained so that the oscillation mode with such a period being a high order $g$-mode of the whole Sun is one of the lowest modes of its core where driving of this oscillation occurs. Further observations would help to verify and specify our ideas about the internal structure of the Sun.

\section{References}

Brookes, J. R., Isaak, G. R., and van der Raay, H. B.: 1976, Nature 259, 92.

Christensen-Dalsgaard, J., Dilke, F. W. W., and Gough, D. O.: 1974, Monthly Notices Roy. Astron. Soc. $169,429$.

Davis, R., Jr.: 1978, Proc. Inform. Conf. Brookhaven Nat. Lab., Upton, New York, Vol. 1, p. 1.

Dilke, F. W. W. and Gough, D. O.: 1972, Nature 240, 262.

Gavryuseva, E. A.: 1981, Soviet Physics - Lebedev Institute Report 10, 52.

Gavryuseva, E. A. and Kopysov, Yu. S.: 1981, Astron. Zh. 58, 610.

Gavryuseva, E. A., Kopysov, Yu. S., and Zatsepin, G. T.: 1981, Soviet Physics - Lebedev Institute Report $10,46$.

Kotov, V. A., Severny, A. B., and Tsap, T. T.: 1976, Nature 259, 87.

Severny, A. B., Kotov, V. A., and Tsap, T. T.: 1979, Astron. Zh. 56, 1137.

Zatsepin, G. T., Gavryuseva, E. A., and Kopysov, Yu. S.: 1980, Rep. Acad. Sci. USSR 251, 1342. 